man ganz kaltes Wasser zur Darstellung des Tropfens verwendet, was wohl gegen die von Colley S. 139 und 140 gegebenen Gründe spricht.

\title{
XI. Nachträglicher Zusatz zu der Abhandlung über die Aberration ${ }^{1}$ ); von E. Ketteler.
}

Die neuerdings von Mascart (Ann. de l'École Norm. No. 3 et 4, 1872) constatirte Unveränderlichkeit der Interferenzstreifen einer parallel zur Axe geschnittenen Krystallplatte im polarisirten (irdischen oder Sonnen-) Licht lărst sich in doppelter Weise ableiten. Man geht entweder von der absoluten Geschwindigkeit der einzelnen Erschütterung aus, oder man berincksichtigt die Länge der inneren Wellen. Ich will zunăchst den ersteren Weg einschlagen und mich überhaupt darauf beschränken, dafs die Richtung des normal einfallenden Lichtes mit der Bewegungsrichtung der Erde zusammenfällt. Heirst dann $\boldsymbol{L}$ die wirkliche (zu unterscheiden von den beiden scheinbaren) Dicke der Krystallplatte, und bezeichnet man die Zeitdifferenz, um die der ordinäre Strahl dem extraordinăren vorauseilt, mit $\Delta$, so hat man:

$$
\Delta=\frac{L\left(1+\frac{g}{\omega_{1}}\right)}{\omega_{1}+g k_{1}}-\frac{L\left(1+\frac{g}{\omega_{1}}\right)}{\omega_{1}+g k_{1}}-\frac{L\left(\frac{g}{\omega_{1}}-\frac{g}{\omega_{2}}\right)}{v} .
$$

Ist noch $T$ die Schwingungsdauer der angewandten terrestrischen oder festen (Fixstern-) Lichtquelle, so hat man für die erstere:

$\Delta=m T\left(1-\frac{g}{v}\right)$, und schliefslich: $L\left(n_{1}-n_{2}\right)=m \cdot v . T$ und fur die $z$ weite:

$$
J=m T, \quad \text { folglich: } L\left(n_{1}-n_{2}\right)\left(1-\frac{g}{v}\right)=m . v . r .
$$

1) In diesem Hefte S. 404. 
Was sodann den anderen Weg, namlich die Bertacksichtigung der inneren Wellen, betrifft, so zeigte ich $§ .28$., dafs far Fixsternlicht:

$$
\lambda_{i}=\lambda^{\prime}\left(1-\frac{g}{v} \frac{n-1}{n}\right)=v^{\prime} T\left(1+\frac{g}{v} \frac{n-1}{n}\right)
$$

ist. Bei Anwendung von irdischem Licht dagegen hat man zunăchst fur einen zwischen Lichtquelle und Mittel gelegenen Punkt des rubenden Aethers:

$$
T_{1}=T\left(1-\frac{g}{v}\right) \text { und darum: } \lambda_{i}=v^{\prime} T_{1}\left(1+\frac{g}{v} \frac{n-1}{n}\right),
$$

folglich:

$$
\lambda_{i}=\lambda^{\prime}\left(1+\frac{g}{v} \frac{1}{n}\right) .
$$

Auf jeden Fall kommen die genannten Wellenlăngen dem ganzen Innern des Mittels, also ebensowohJ den transferirten als den nicht transferirten Theilchen desselben zu. Es entspricht also einer Länge $L$ des Mittels eine Anzahl von Wellenlängen, die gleich ist resp. $\frac{L}{\left(\lambda_{1}\right)_{1}}$ und $\frac{L}{\left(\lambda_{1}\right)_{9}}$.

So kommt zunächst för irdisches Licht:

oder

$$
\frac{L}{\lambda}\left(\frac{n_{1}}{1+\frac{g}{v} \frac{1}{n_{1}}}-\frac{n_{2}}{1+\frac{g}{v} \frac{1}{n_{2}}}\right)=m
$$

$$
L\left(n_{1}-n_{2}\right)=m \lambda=m .0 . T .
$$

Und fur Fissternlicht:

oder:

$$
\frac{L}{\lambda}\left(\frac{n_{1}}{1+\frac{g}{v} \frac{n_{1}-1}{n_{1}}}-\frac{n_{1}}{1+\frac{g}{v} \frac{n_{2}-1}{n_{1}}}\right)=m
$$

$$
L\left(n_{1}-n_{2}\right)\left(1-\frac{g}{v}\right)=m \lambda
$$

Hr. Mascart dagegen deutet das von ihm angestellte Experiment als in Widerspruch stehend mit der Fresnel'schen Formel. Im Uebrigen giebt derselbe in der angesogenen Arbeit eine Ansahl oon Rechnungen und Sätsen, die ich bereits nicht blo/s mehrere Monate frither, sondern auch sehr viel allyemeiner in diesen Annalen (Bd. 144) veröffentlickt habe. 